\title{
State and Trait Anxiety Scores of Patients Receiving Intravitreal Injections
}

\author{
Ozcan Kayikcioglu $^{a}$ Sinan Bilgin ${ }^{c}$ Goktug Seymenoglu ${ }^{a}$ \\ Artuner Deveci ${ }^{\mathrm{b}}$ \\ Departments of a Ophthalmology and ${ }^{\mathrm{b}}$ Psychiatry, Hafsa Sultan Hospital, \\ Faculty of Medicine, Celal Bayar University, Manisa, and 'Department of Ophthalmology, \\ Medifema Hospital, Izmir, Turkey
}

\begin{abstract}
What Is It about?
Intravitreal anti-vascular endothelial growth factor injections are widely used all over the world. More than one injection may be usually required for treatment. The number of injections and other factors may increase anxiety in patients. This situation may affect their general comfort and decrease compliance with recurring injections. This study was conducted to assess the effects of various parameters on patient anxiety.
\end{abstract}

\section{Keywords}

Bevacizumab · Intravitreal injection · Ranibizumab · Status anxiety scores · Trait anxiety scores

\section{Abstract}

Background: To evaluate parameters on the state and trait anxiety scores of patients receiving intravitreal injections. Methods: One hundred thirteen patients were included in the study. All subjects received intravitreal ranibizumab or bevacizumab injections. To measure the level of anxiety, Spielberg's State-Trait Anxiety Inventory questionnaire was used. Results: The mean state anxiety scores were $45.19 \pm 5.62$ in experienced patients and $43.10 \pm 6.62$ in inexperienced patients $(p=0.078)$. The mean trait anxiety scores were $50.14 \pm 6.62$ in experienced patients and $49.17 \pm 10.79$ in inexperienced patients $(p=0.810)$. Additionally, there was no statistically significant difference in the state and trait anxiety scores between the male and female, employed, and retired patients $(p>0.05)$. Conclusion: Anxiety may not show significant differences according to sociodemographic status. High anxiety scores found in this study also emphasize that health care providers should try to decrease anxiety levels during the course of treatment. 


\section{Introduction}

Intravitreal anti-vascular endothelial growth factor injections are used in the treatment of various diseases, including diabetic retinopathy, age-related macular degeneration, neovascular glaucoma, and intraocular inflammation. More than one injection may be usually required for the management of these diseases. On the other hand, patient opinion about a drug injection into the eye may stimulate much anxiety and may even lead to much pain. This situation may affect their general comfort and decrease compliance with recurring injections [1]. Therefore, the aim of this study was to assess the effects of various parameters, mainly previous experience with intravitreal injection on patient anxiety.

\section{Methods}

This prospective, consecutive, observational, noninterventional study included 113 patients with diagnosis of wet-type age-related macular degeneration (AMD) and diabetic macular edema (DME). None of the patients had known psychiatric conditions or used anxiolytic drugs. All subjects received intravitreal ranibizumab or bevacizumab injections performed by the same surgeon. To measure the level of anxiety, Spielberg's State-Trait Anxiety Inventory (STAI) questionnaire was used. All patients completed the questionnaire by themselves immediately before intravitreal injection.

STAI is the "gold standard" for measuring preoperative anxiety [2-4]. It comprises separate self-report scales for measuring two distinct anxiety concepts: state anxiety and trait anxiety. The reliability and validity of the STAI are well reported (Cronbach's alpha = 0.896). The STAI-T scale consists of 20 statements that ask people to describe how they generally feel. The STAI-S scale also consists of 20 statements, but the instructions require subjects to indicate how they feel at a particular moment in time. The STAI-S scale can be used to determine the actual levels of anxiety intensity induced by stressful procedures. The validity of the STAI rests upon the assumption that the examinee has a clear understanding of the "state" and "trait" instructions. Each question is rated on a 4-point scale (not at all, somewhat, moderately so, very much so). The range of possible scores for form Y of the STAI varies from a minimum score of 20 to a maximum score of 80 on both the STAI-T and STAI-S subscales. STAI scores are commonly classified as "no or low anxiety" (20-37), "moderate anxiety" (38-44), and "high anxiety" (45-80). We used form Y of STAI in English and had it translated into Turkish by an expert in the respective languages. The translated forms were then retranslated back to English. The retranslated sentences which most closely resembled the original English STAI form Y sentences were used for that language.

SPSS, version 18 (SPSS Inc., Chicago, IL, USA) was used for statistical analysis. Normality of the data was tested with a Kolmogorov-Smirnov test to indicate the appropriateness of parametric testing.

Values are presented as means \pm standard deviation. A Mann-Whitney U test and Wilcoxon test were used for nonparametric data. Parametric data were analyzed using a Student $t$ test. Pearson correlation and Spearman correlation tests were used to measure the linear association between two variables. A $p$ value of less than 0.05 was considered to be statistically significant. 
Table 1. Results of state and trait anxiety scores in different parameters

\begin{tabular}{lll}
\hline Parameters & State anxiety scores & $p$ value \\
\hline Experience (Y vs. N) & $45.19 \pm 5.62$ vs. $43.10 \pm 6.62$ & 0.078 \\
Gender (M vs. F) & $45.18 \pm 6.54$ vs. $43.67 \pm 5.49$ & 0.186 \\
Employed vs. retired & $44.73 \pm 8.86$ vs. $44.33 \pm 5.21$ & 0.783 \\
Diagnosis (AMD vs. DME) & $45.08 \pm 5.57$ vs. $43.26 \pm 6.68$ & 0.122 \\
\hline Parameters & Trait anxiety scores & $p$ value \\
\hline Experience (Y vs. N) & $50.14 \pm 6.62$ vs. $49.17 \pm 10.79$ & 0.810 \\
Gender (M vs. F) & $49.33 \pm 7.71$ vs. $50.21 \pm 8.97$ & 0.434 \\
Employed vs. retired & $49.50 \pm 8.72$ vs. $49.85 \pm 8.32$ & 0.606 \\
Diagnosis (AMD vs. DME) & $51.07 \pm 7.78$ vs. $47.60 \pm 8.93$ & $0.033^{*}$ \\
\hline Correlation between parameters & & \\
Age/state anxiety scores & & $p=0.145, r=0.138$ \\
Age/trait anxiety scores & & $p=0.236, r=0.112$ \\
\hline
\end{tabular}

Y, yes; N, no; AMD, age-related macular degeneration; DME, diabetic macular edema. * Statistically significant difference.

\section{Results}

Out of 113 patients, $58(51.3 \%)$ were female and 55 (48.6\%) were male. The mean age of the participants was $67.02 \pm 9.5$ years. Ninety-one patients $(80.5 \%)$ were retired during the study period while $22(19.4 \%)$ were in active working life. Seventy-one patients $(63.7 \%)$ had a diagnosis of AMD and 42 (37.1\%) had a diagnosis of DME. Seventy-one patients (63.7\%) had experience with intravitreal injections because they had received at least one intravitreal injection previously, and 42 inexperienced patients (37.1\%) received an intravitreal injection for the first time. The average state and trait anxiety scores were $49.78 \pm 8.36$ and $44.41 \pm$ 6.04 , respectively (Wilcoxon test $p<0.001$ ).

There was a weakly positive but nonsignificant relationship between age and state and trait anxiety scores (Pearson correlation, $r=0.138, p=0.145$ and Spearman's correlation $r=$ $0.112, p=0.236$ respectively).

Experienced, employed, male patients had higher state anxiety scores than inexperienced, retired, female patients, but the difference did not reach statistical significance (Student's $t$ test, $p=0.783, p=0.078, p=0.186$, respectively) (Table 1).

Furthermore, experienced, employed, male patients had higher trait anxiety scores than inexperienced, retired, female patients, but the difference did not reach statistical significance (Mann-Whitney U test, $p=0.810, p=0.606, p=0.434$, respectively).

However, patients with AMD had higher anxiety scores than DME patients, and the difference in trait anxiety scores between groups was statistically significant $(p=0.033)$.

\section{Discussion}

Intravitreal injection is one of the most common treatments in most tertiary eye care centers. In recent application protocols, patients should receive repetitive injections during the treatment process. Although repetitive injections are an economical and psychological burden for the patients, they are necessary for treatment success. 
Segal et al. [5] showed that one quarter of the patients in their study had high anxiety scores. They also found significant positive correlation between pain during the injection and preprocedural anxiety level. In their study, retired patients and male gender had higher pain scores than patients who were employed and female. Therefore, we expected that anxiety level would be higher in retired and male patients, but we did not find a significant difference between employed and retired and between male and female. Furthermore, Segal et al. did not find a significant correlation between pain score and prior injections. In the current study, being in employment, gender, age, and previous experience with intravitreal injection were not significantly associated with anxiety levels. Increased experience did not resolve patient anxiety, which might be due to previously perceived pain or the idea of a continuing disease process. Although we expected that younger patients would have higher anxiety scores, age had an unexpected positive correlation with anxiety scores.

Similarly, in a study conducted by Chen et al. [6] it was reported that there was no statistically significantrelation between anxiety and the number of previous injections. Furthermore, they proposed that listening to classical music before and during intravitreal injections significantly decreased anxiety in patients.

Also, in this study, the average state anxiety score was significantly higher than trait anxiety scores. This difference can be associated with having an intravitreal injection procedure, but can also be observed when patients attend hospital or see doctors ("white coat" phenomenon).

Moreover, fear of job loss, vision loss, and struggle to earn a living may be other possible reasons for increased state anxiety in employed patients.

Segal et al. [5] evaluated pain scores in different diagnoses, AMD versus DME, and found patients with AMD had higher pain scores than DME patients. The difference of anxiety level between the two diseases was not evaluated in their study. In our study, it was found that AMD patients had higher anxiety scores. In looking at the results of our study and the one by Segal et al., it may be said that the injection process affected AMD patients more than DME patients. This situation may point to these two different diseases having their own psychological and pathophysiological processes. In order to understand the differences between the two diseases, social and economic situations in addition to the processes described above should be investigated.

In conclusion, anxiety is a crucial issue for all patients receiving an intravitreal injection, and it may not show significant differences according to sociodemographic status. AMD and diabetes mellitus may be considered two different diseases which have their own psychological and pathophysiological processes. The relevance of the results would be much greater if we had an intraindividual comparison of injections between inexperienced and experienced patients. Nevertheless, high anxiety scores found in this study emphasize that health care providers should try to decrease anxiety levels during the course of treatment.

\section{Statement of Ethics}

The study adhered to the principles of the Declaration of Helsinki. Informed consent was obtained from all participants.

\section{Disclosure Statement}

The authors have declared that no competing financial interests exist. 
(c) 2017 The Author(s). Published by S. Karger AG, Basel www.karger.com/bmh

Kayikcioglu et al.: State and Trait Anxiety Scores of Patients Receiving Intravitreal Injections

\section{References}

1 Cintra LP, Lucena LR, Da Silva JA, Costa RA, Scott IU, Jorge R: Comparative study of analgesic effectiveness using three different anesthetic techniques for intravitreal injection of bevacizumab. Ophthalmic Surg Lasers Imaging 2009;40:13-18.

-2 Spielberger CD, Lushene RE, Jacobs GA: Manual for the State-Trait Anxiety Inventory, STAI (Form Y). Palo Alto, Consulting Psychologists Press, 1983.

-3 Nigussie S, Belachew T, Wolancho W: Predictors of preoperative anxiety among surgical patients in Jimma University Specialized Teaching Hospital, South Western Ethiopia. BMC Surg 2014;14:67.

4 Dalal KS, Chellam S, Toal P: Anaesthesia information booklet: is it better than a pre-operative visit? Indian J Anaesth 2015;59:511-513.

-5 Segal O, Segal-Trivitz Y, Nemet AY, Cohen P, Geffen N, Mimouni M: Anxiety levels and perceived pain intensity during intravitreal injections. Acta Ophthalmol 2016;94:203-204.

-6 Chen X, Seth RK, Rao VS, Huang JJ, Adelman RA: Effects of music therapy on intravitreal injections: a randomized clinical trial. J Ocul Pharmacol Ther 2012;28:414-419. 